\title{
Adherence to the Dietary Approaches to Stop Hypertension (DASH) diet in relation to obesity among Iranian female nurses
}

\author{
Farzaneh Barak ${ }^{1,2}$, Ebrahim Falahi ${ }^{3}$, Ammar Hassanzadeh Keshteli ${ }^{4}$, \\ Ahmadreza Yazdannik ${ }^{5}$ and Ahmad Esmaillzadeh ${ }^{1,2, *}$ \\ ${ }^{1}$ Food Security Research Center, Isfahan University of Medical Sciences, Isfahan, Islamic Republic of Iran: \\ ${ }^{2}$ Department of Community Nutrition, School of Nutrition and Food Science, Isfahan University of Medical Sciences, \\ Isfahan, PO Box 81745-151, Islamic Republic of Iran: ${ }^{3}$ Department of Nutrition, Faculty of Health and Nutrition, \\ Lorestan University of Medical Sciences, Khorramabad, Islamic Republic of Iran: ${ }^{4}$ Integrative Functional \\ Gastroenterology Research Center, Isfahan University of Medical Sciences, Isfahan, Islamic Republic of Iran: \\ ${ }^{5}$ Department of Critical Care Nursing, School of Nursing and Midwifery, Isfahan University of Medical Sciences, \\ Isfahan, Islamic Republic of Iran
}

Submitted 26 November 2012: Final revision received 18 December 2013: Accepted 3 April 2014: First published online 8 May 2014

\begin{abstract}
Objective: Limited observational studies have considered habitual consumption of the general population to examine the relationship between the Dietary Approaches to Stop Hypertension (DASH) diet and obesity. The aim of the present study was to investigate adherence to the DASH diet in relation to general and central obesity among female nurses in Isfahan, Iran.

Design: Cross-sectional study carried out among 293 female nurses aged $>30$ years who were selected by a multistage, cluster random sampling method. Usual dietary intakes were assessed using a validated FFQ. We constructed the DASH score based on foods and nutrients emphasized or minimized in the DASH diet, focusing on eight components: high intake of fruits, vegetables, nuts and legumes, low-fat dairy products and whole grains and low intakes of sodium, sweetened beverages, and red and processed meats. General and abdominal obesity were defined as BMI $\geq 25 \mathrm{~kg} / \mathrm{m}^{2}$ and waist circumference $\geq 88 \mathrm{~cm}$, respectively.

Setting: Isfahan, Iran.

Subjects: Female nurses ( $n$ 293) aged $>30$ years.

Results: Increased adherence to the DASH diet was associated with older age $(P<0.01)$ and lower waist circumference $(P=0.04)$. There was no statistically significant difference in the prevalence of general obesity between extreme quartiles of the DASH diet score. After adjustment for age, energy intake and other confounding factors, DASH diet score was not significantly associated with obesity. However, with further controlling for other dietary factors, those in the highest quartile of DASH diet score were $71 \%$ less likely to have general obesity compared with those in the lowest quartile. In addition, following a DASH diet was inversely associated with central obesity after adjustment for potential confounders ( $\mathrm{OR}=0 \cdot 37 ; 95 \% \mathrm{CI} 0 \cdot 14,0 \cdot 96)$.

Conclusions: We found that adherence to the DASH diet was inversely related to central obesity among Iranian adult females. This association remained significant even after adjustment for potential confounders.
\end{abstract}

The prevalence of overweight and obesity is rapidly increasing worldwide. During the past decades, obesity and obesity-related co-morbidities have become epidemic in the $\operatorname{world}^{(1)}$. The worldwide prevalence of obesity nearly doubled between 1980 and $2008^{(2)}$ and it has been estimated that in 2030 , globally an estimated $2 \cdot 16$ billion adults will be overweight and 1.12 billion will be obese ${ }^{(3)}$. Epidemiological studies have shown that obesity, and in particular central obesity, predicts the risk of developing hypertension, diabetes, cardiovascular mortality and proinflammatory conditions ${ }^{(4)}$. It has been estimated that $21 \%$ of cardiovascular events in men and $28 \%$ in women can be explained by overweight and obesity ${ }^{(5)}$.

With improvement in the socio-economic situation in developing countries, an increasing prevalence of obesity is observed among both adults and children in these countries $^{(6)}$. This is particularly true in the Middle East and North Africa and in Latin America and the Caribbean ${ }^{(7)}$. 
A recent systematic review of published data from the Middle Eastern and North African countries indicated an alarming level of obesity prevalence among women $(>40 \%)$ in the oil-rich countries ${ }^{(8)}$. The prevalence of overweight and obesity in Iran was found to be $42.8 \%$ in men and $57.0 \%$ in women in $2005^{(9)}$. The prevalence of abdominal obesity in Iranian women and men was reported to be $54.5 \%$ and $12.9 \%$, respectively ${ }^{(9)}$. Recent estimates show that the prevalence of obesity in Iran is seemingly increasing at an alarming rate ${ }^{(10)}$. A significant change in lifestyle and dietary habits in recent decades has been suggested to play an important role in the increasing obesity prevalence in Middle Eastern and North African countries. There has been a consistent rise in per capita energy supply, mostly coming from animal foods, increased consumption of saturated fat and refined carbohydrates and decreased intake of fresh fruits and vegetables $^{(8)}$.

Diet has long been the subject of intense scientific research on obesity. Due to the co-linearity of nutrients and foods, the dietary pattern approach has attracted considerable interest in the field of obesity research ${ }^{(11)}$. The dietary pattern approach considers the role of the whole diet in predicting risk of chronic diseases ${ }^{(12)}$. The Dietary Approaches to Stop Hypertension (DASH) eating plan is an a priori-defined dietary pattern recommended by the US Department of Agriculture's dietary guidelines in 2005 as a healthy dietary pattern for adults ${ }^{(13)}$. Although this dietary pattern was initially suggested for the treatment of hypertension ${ }^{(14)}$, its favourable effect on diabetes $^{(15,16)}$, the metabolic syndrome ${ }^{(17)}$ and $\mathrm{CVD}^{(18)}$ has been indicated. The DASH diet consists mainly of high amounts of fruits, vegetables, nuts and legumes, low-fat dairy products and whole grains and low amounts of sodium, sweetened beverages, and red and processed meats. Most of these components have been shown to influence insulin resistance, metabolic disorders and inflammation $^{(19)}$. It has also been indicated to reduce the risk of developing type 2 diabetes, so that in a large cohort study among men, those in the top quintile of the DASH score had a $25 \%$ lower risk than those in the bottom quintile ${ }^{(20)}$. Earlier studies have also shown that adherence to the DASH diet favourably affects weight control $^{(17,21,22)}$. Azadbakht et al. ${ }^{(17)}$ showed that following a DASH diet could lead to decreased body weight and waist circumference in patients with metabolic syndrome. They also reported similar findings in another dietary intervention study on type 2 diabetes mellitus patients ${ }^{(21)}$. Furthermore, Moore et al. showed that participating in an Internet-based nutrition education programme on the DASH diet could result in significantly reduced body weight after 12 months ${ }^{(22)}$.

Observational studies regarding associations between the DASH diet and health conditions have focused mainly on $\mathrm{CVD}^{(18,19)}$, cancers ${ }^{(23)}$ and other chronic diseases ${ }^{(15,16)}$. Although the potential role of the DASH diet in weight management has been shown in a number of studies mainly on diseased populations, the relationship between adherence to the DASH diet and obesity in the general population has been investigated in only a limited number of observational studies to date. The previous observational studies in this regard were carried out among selected groups of people, such as diabetics, which further restricts their generalizability. For instance, it has been indicated that prevalence of obesity was not associated with adherence to the DASH diet among type 1 or type 2 diabetic patients ${ }^{(15)}$.

Studying the relationship between the DASH diet and general and central obesity is particularly relevant for Middle Eastern countries due to the unique characteristics of dietary intakes in this region: large portion sizes with high intakes of refined grains (white rice and bread) and hydrogenated fats and a greater percentage of energy from carbohydrates, in addition to lack of alcohol use. The Iranian dietary pattern includes high intakes of refined grains, potato, tea, hydrogenated fats, legumes and broth $^{(24)}$.

To our knowledge, no previous study has examined the relationship between adherence to the DASH dietary pattern and general and central adiposity, particularly in a Middle Eastern setting. The present study was carried out to investigate DASH diet adherence in relation to general and central obesity among female nurses in Isfahan, Iran.

\section{Participants and methods}

\section{Participants}

The current cross-sectional study was carried out among a representative sample of Isfahani female nurses aged $>30$ years who were selected by a multistage, cluster random sampling method. Samples were randomly selected from seven hospitals, of them five were public (Kashani, Alzahra, Beheshti, Chamran and Khorshid) and two were private hospitals (Sadi and Isfahan Clinic). In total, 420 nurses agreed to take part in the study. Informed written consent was obtained from each participant. We excluded those with reported energy intake outside the range of $3347-18828 \mathrm{~kJ} / \mathrm{d}(800-4500 \mathrm{kcal} / \mathrm{d})$ as well as those with a chronic disease affecting their diet. We also excluded those who had been following a special dietary regimen in the last year (e.g. weight-loss diet). After these exclusions, 293 participants remained for the current analysis.

\section{Assessment of dietary intakes}

Usual dietary intakes were assessed using a 106-item, selfadministered, dish-based, semi-quantitative FFQ (DFQ) ${ }^{(25)}$. The DFQ was designed to capture information on dietary intakes of Iranian adults over the preceding year. Foods and dishes were classified into five main domains such as: (i) mixed dishes; (ii) grains; (iii) dairy products; (iv) fruits 
and vegetables; and (v) miscellaneous food items and beverages. Participants were asked to mention their consumption frequency of a specified portion of each food or dish item during the previous year. The frequency response section consisted of multiple choices and ranged from 'never or less than once a month' to 'twelve or more times per day'. The number of frequency response categories was not similar for all foods, as those food items that were consumed more frequently had more frequency response categories. The DFQ was validated among a sample of 200 randomly selected Isfahani adults. The reliability of the DFQ was assessed by comparing dietary intakes estimated by responses to the DFQ on two different occasions. The validity of the DFQ was assessed using three $24 \mathrm{~h}$ dietary recalls. Overall, these data indicated that the DFQ provides reasonably valid and reliable measures of the average long-term dietary intakes.

\section{Adherence to the DASH dietary pattern}

We constructed the DASH score based on foods and nutrients emphasized or minimized in the DASH diet, focusing on eight components: high intake of fruits, vegetables, nuts and legumes, low-fat dairy products and whole grains, as well as low intakes of sodium, sweetened beverages, and red and processed meats ${ }^{(19)}$. We calculated a DASH score for each study participant. First, participants were classified based on quartile categories of consuming the previously mentioned food items. For fruits, vegetables, nuts and legumes, low-fat dairy products and whole grains, those in the first (lowest) dietary intake quartile were given the score of 1 and those in the fourth (highest) quartile were given the score of 4 . Other quartiles (second and third) for these dietary intakes were given the corresponding score (2 and 3 , respectively). For sodium, red and processed meats, and sweetened beverages, the lowest quartile of dietary intake was given a score of 4 and the highest quartile was given the score of 1 . Those in the third and second quartiles for these dietary intakes were given the score of 2 and 3 , respectively. Since dietary sodium intake cannot be assessed properly using an FFQ, the current scoring by means of quartiles would be least prone to misclassification. The scores were then summed up to construct the overall adherence to the DASH diet score that ranged from 8 to 32; individuals with the highest DASH score were more likely to follow the DASH diet.

\section{Assessment of anthropometric measures}

Weight was measured to the nearest $100 \mathrm{~g}$ without shoes while wearing minimal clothes. Height was measured without shoes with shoulders in a normal position. BMI was calculated as weight in kilograms divided by the square of height in metres. In the current study, general obesity was defined as BMI $\geq 25 \mathrm{~kg} / \mathrm{m}^{2}$. Waist circumference was measured at the narrowest level and hip circumference at the maximum level over light clothing using a non-stretchable tape measure, without any pressure to the body surface. Measurements were recorded to the nearest $0.1 \mathrm{~cm}$. We defined central obesity as waist circumference $\geq 88 \mathrm{~cm}$.

\section{Assessment of other variables}

Daily physical activity was assessed through the short form of the International Physical Activity Questionnaire ${ }^{(26)}$ and was expressed as MET-h/week (where MET = metabolic equivalent of task). Additional covariate information regarding age, smoking habits, socio-economic status, marital status, menopausal situation, medical history, and current use of medications and supplements was obtained using a self-administered questionnaire. Socio-economic status was defined based on educational level, income, family size, being the owner of one's house or renting the house, house area, being the owner of a car and number and kind of car, number of bedrooms and determination of who was in charge of the family.

\section{Statistical methods}

Participants were categorized based on the quartiles of DASH dietary pattern scores. To compare general characteristics across quartiles, we used one-way ANOVA and $\chi^{2}$ tests where appropriate. Dietary intakes (ageand energy-adjusted) were compared using ANCOVA. Multivariate-adjusted means for anthropometric measures were computed using a general linear model in different models controlling for age (years) and energy intake $(\mathrm{kcal} / \mathrm{d})$ in model I; current use of oral contraceptive pills (yes or no), current use of corticosteroids (yes or no), physical activity (MET-h/week), marital status (married or not married), menopausal status (yes or no) and socioeconomic status (low/medium/high) in model II; and additionally for dietary intakes of refined grains, sweets, white meat, oils, high-fat dairy products and eggs in model III. To investigate the relationship between DASH dietary pattern and risk of general and central obesity, we used multivariate logistic regression. Covariates were the same as above. All statistical analyses were done using the statistical software package PASW Statistics version 18. $P$ values less than 0.05 were considered to be statistically significant.

The study protocol was approved the Regional Biomedical Ethics Committee affiliated to Isfahan University of Medical Sciences.

\section{Results}

Baseline characteristics of study participants across quartiles of DASH scores are presented in Table 1. Increased adherence to the DASH diet was associated with older age $(P<0.01)$ and lower waist circumference $(P=0.04)$. Consumption of the DASH dietary pattern was not significantly associated with weight, BMI or hip circumference. The distribution of individuals in terms of menopausal 
Table 1 Baseline characteristics according to quartile of Dietary Approaches to Stop Hypertension (DASH) diet score among female nurses ( $n$ 293) aged $>30$ years in Isfahan, Iran

\begin{tabular}{|c|c|c|c|c|c|c|c|c|c|}
\hline & \multicolumn{8}{|c|}{ Quartile of DASH score } & \multirow[b]{3}{*}{$P^{*}$} \\
\hline & \multicolumn{2}{|c|}{ Q1 } & \multicolumn{2}{|c|}{ Q2 } & \multicolumn{2}{|c|}{ Q3 } & \multicolumn{2}{|c|}{ Q4 } & \\
\hline & Mean & SD & Mean & SD & Mean & SD & Mean & SD & \\
\hline Age (years) & 33.6 & $7 \cdot 0$ & $32 \cdot 7 \dagger$ & $6 \cdot 8$ & $36 \cdot 0$ & 6.9 & $35 \cdot 6$ & 6.5 & 0.01 \\
\hline Weight (kg) & $62 \cdot 3$ & 9.1 & $61 \cdot 1$ & $10 \cdot 2$ & 63.8 & 9.0 & $61 \cdot 1$ & 8.2 & 0.32 \\
\hline BMI $\left(\mathrm{kg} / \mathrm{m}^{2}\right)$ & 23.9 & 3.5 & 23.3 & 3.3 & 24.4 & 3.6 & $23 \cdot 6$ & 3.0 & 0.35 \\
\hline Waist circumference $(\mathrm{cm})$ & 80.9 & 9.5 & 78.4 & $10 \cdot 1$ & $82 \cdot 3$ & $10 \cdot 1$ & $78 \cdot 2$ & 8.3 & 0.04 \\
\hline Hip circumference $(\mathrm{cm})$ & $100 \cdot 1$ & 7.6 & 99.0 & $9 \cdot 3$ & 101.5 & $7 \cdot 1$ & $99 . \overline{6}$ & 7.4 & 0.39 \\
\hline \multirow[t]{2}{*}{ Physical activity (MET-h/week) } & $80 \cdot 4$ & $100 \cdot 7$ & $69 \cdot 0$ & $90 \cdot 1$ & $84 \cdot 7$ & $84 \cdot 0$ & $70 \cdot 7$ & $71 \cdot 8$ & 0.84 \\
\hline & \multicolumn{2}{|c|}{$\%$} & \multicolumn{2}{|c|}{$\%$} & \multicolumn{2}{|c|}{$\%$} & \multicolumn{2}{|c|}{$\%$} & \\
\hline Current oral contraceptive pill users (\%) & \multicolumn{2}{|c|}{$6 \cdot 8$} & \multicolumn{2}{|c|}{$12 \cdot 2$} & \multicolumn{2}{|c|}{0} & \multicolumn{2}{|c|}{8.0} & 0.07 \\
\hline Current corticosteroid users (\%) & \multicolumn{2}{|c|}{$1 \cdot 1$} & \multicolumn{2}{|c|}{0} & \multicolumn{2}{|c|}{0} & \multicolumn{2}{|c|}{0} & 0.51 \\
\hline Menopausal status (\%) & \multicolumn{2}{|c|}{4.5} & \multirow{2}{*}{\multicolumn{2}{|c|}{$\begin{array}{r}5.4 \\
25.5\end{array}$}} & \multicolumn{2}{|c|}{$3 \cdot 8$} & \multicolumn{2}{|c|}{$1 \cdot 3$} & 0.58 \\
\hline High socio-economic statusł (\%) & \multirow{2}{*}{\multicolumn{2}{|c|}{$\begin{array}{l}23 \cdot 7 \\
73 \cdot 0\end{array}$}} & & & \multirow{2}{*}{\multicolumn{2}{|c|}{$\begin{array}{l}40.0 \\
76.9\end{array}$}} & \multirow{2}{*}{\multicolumn{2}{|c|}{$\begin{array}{l}33 \cdot 3 \\
71 \cdot 1\end{array}$}} & 0.60 \\
\hline Married (\%) & & & \multicolumn{2}{|c|}{$\begin{array}{l}25 \cdot 5 \\
64.9\end{array}$} & & & & & 0.49 \\
\hline
\end{tabular}

MET, metabolic equivalent of task.

${ }^{*}$ Obtained from ANOVA for continuous variables and $\chi^{2}$ test for categorical variables.

†Mean value was significantly different from that in the third and fourth quartiles by paired $t$-test $(P<0.05)$.

fHigh socio-economic status was defined based on educational level, income, family size, being the owner of one's house or renting the house, house area, being the owner of a car and number and kind of car, number of bedrooms and determination of who was in charge of the family.

Table 2 Dietary intakes according to quartile of Dietary Approaches to Stop Hypertension (DASH) diet score among female nurses $(n 293)$ aged $>30$ years in Isfahan, Iran*

\begin{tabular}{|c|c|c|c|c|c|c|c|c|c|}
\hline & \multicolumn{8}{|c|}{ Quartile of DASH score } & \multirow[b]{3}{*}{$P+$} \\
\hline & \multicolumn{2}{|c|}{ Q1 } & \multicolumn{2}{|c|}{ Q2 } & \multicolumn{2}{|c|}{ Q3 } & \multicolumn{2}{|c|}{ Q4 } & \\
\hline & Mean & SE & Mean & SE & Mean & SE & Mean & SE & \\
\hline \multicolumn{10}{|l|}{ Food groups } \\
\hline Fruits $(\mathrm{g} / \mathrm{d})$ & 200 & 163 & 332 & 309 & 480 & 310 & 579 & 327 & $<0.001$ \\
\hline Vegetables $(\mathrm{g} / \mathrm{d})$ & 240 & 113 & 279 & 170 & 329 & 128 & 508 & 315 & $<0.001$ \\
\hline Red meat $(\mathrm{g} / \mathrm{d})$ & 157 & 89 & 142 & 94 & 127 & 100 & 128 & 812 & 0.13 \\
\hline Low-fat dairy (g/d) & 210 & 144 & 362 & 304 & 384 & 342 & 473 & 280 & $<0.001$ \\
\hline Sugar-sweetened beverages $(\mathrm{g} / \mathrm{d})$ & 236 & 275 & 166 & 246 & 110 & 152 & 83 & 111 & $<0.001$ \\
\hline Oils $(g / d)$ & 80 & 32 & 68 & 31 & 67 & 35 & 62 & 28 & 0.001 \\
\hline Whole grains $(g / d)$ & 26 & 43 & 65 & 149 & 59 & 96 & 86 & 87 & 0.001 \\
\hline Nuts and legumes $(\mathrm{g} / \mathrm{d})$ & 49 & 30 & 59 & 44 & 58 & 39 & 65 & 33 & 0.05 \\
\hline \multicolumn{10}{|l|}{ Nutrients } \\
\hline Energy (kcal)‡ & 2657 & 761 & 2652 & 921 & 2747 & 834 & 2845 & 769 & 0.41 \\
\hline Protein $(\mathrm{g} / \mathrm{d})$ & 120 & 146 & 102 & 69 & 109 & 83 & 149 & 160 & 0.15 \\
\hline Fat $(g / d)$ & 110 & 33 & 102 & 34 & 101 & 33 & 155 & 126 & 0.57 \\
\hline Cholesterol (mg/d) & 260 & 125 & 241 & 119 & 234 & 106 & 244 & 118 & 0.59 \\
\hline $\mathrm{Na}(\mathrm{mg} / \mathrm{d})$ & 4372 & 1972 & 3828 & 1306 & 3903 & 1832 & 3783 & 1296 & 0.07 \\
\hline $\mathrm{K}(\mathrm{mg} / \mathrm{d})$ & 2881 & 962 & 3193 & 1222 & 3604 & 1179 & 4494 & 1632 & $<0.001$ \\
\hline $\mathrm{Ca}(\mathrm{mg} / \mathrm{d})$ & 744 & 234 & 908 & 430 & 947 & 383 & 716 & 456 & $<0.001$ \\
\hline$M g(m g / d)$ & 221 & 63 & 250 & 92 & 268 & 84 & 324 & 101 & $<0.001$ \\
\hline Folate $(\mathrm{mg} / \mathrm{d})$ & 246 & 79 & 285 & 118 & 312 & 93 & 404 & 176 & $<0.001$ \\
\hline Total dietary fibre $(\mathrm{g} / \mathrm{d})$ & 16 & 5 & 18 & 8 & 22 & 7 & 29 & 25 & $<0.001$ \\
\hline
\end{tabular}

*Nutrients and food intakes have been adjusted for age and total energy intake.

†Obtained from ANCOVA for continuous variables and $\chi^{2}$ test for categorical variables.

$\ddagger 1 \mathrm{kcal}=4 \cdot 184 \mathrm{~kJ}$.

status, socio-economic status, marital status, current use of corticosteroids and current use of oral contraceptive pills was not significantly different across quartile categories of DASH score.

Adherence to the DASH diet was associated with significantly greater intakes of fruits, vegetables, low-fat dairy (all $P<0 \cdot 001)$, whole grains $(P=0 \cdot 001)$, nuts and legumes $(P=0.05 ;$ Table 2$)$. Individuals in the fourth quartile of DASH score had significantly lower intakes of sugar-sweetened beverages $(P<0 \cdot 001)$ and oils $(P=0.001)$ compared with those in the first quartile. There was no statistically significant association between adherence to the DASH diet and total energy or macronutrient intakes. Individuals in the fourth quartile of DASH diet score had 

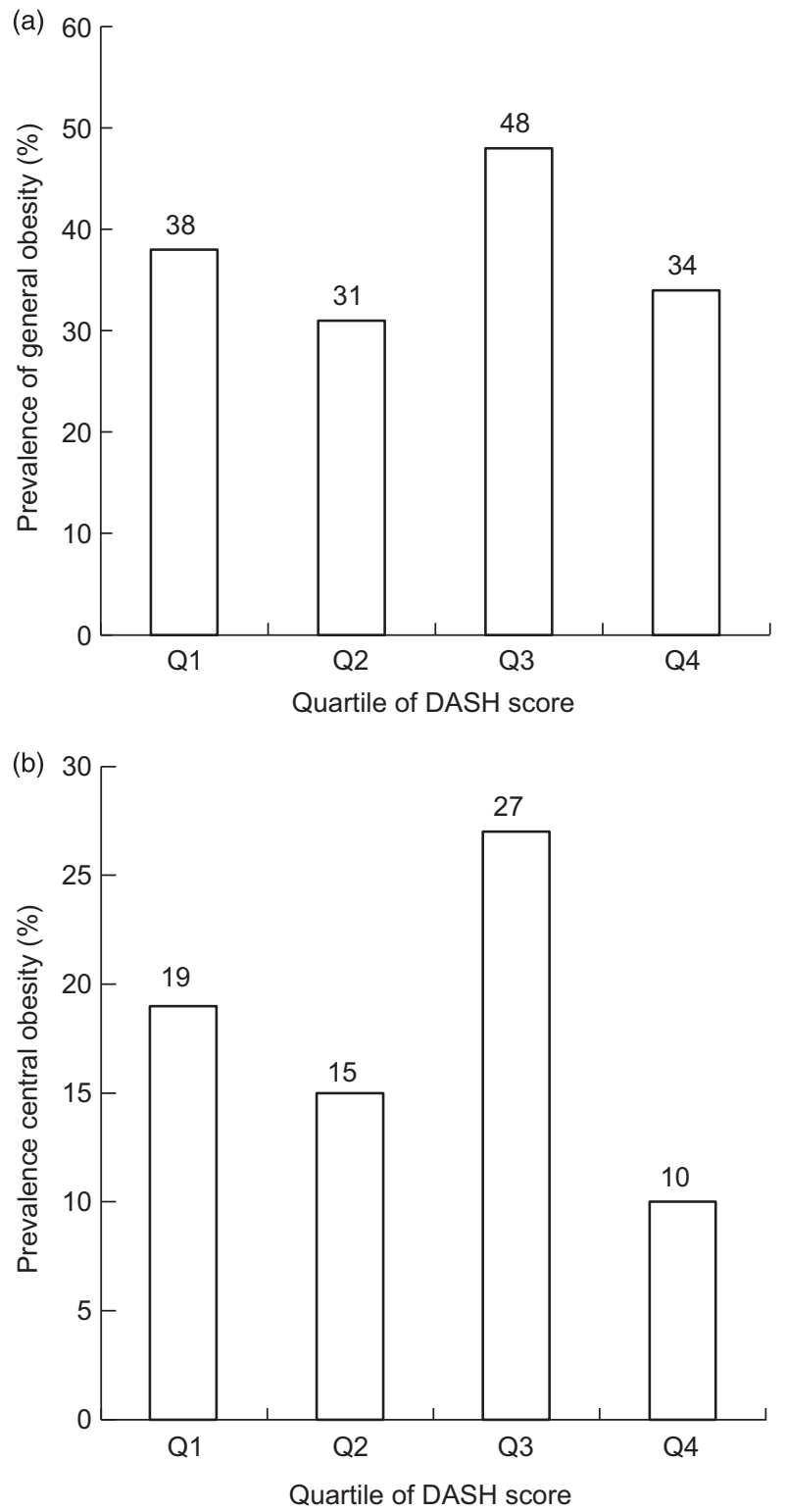

Fig. 1 Prevalence of general (a) and central obesity (b) across quartiles of Dietary Approaches to Stop Hypertension (DASH) diet score among female nurses ( $n$ 293) aged $>30$ years in Isfahan, Iran. There was no statistically significant difference in the prevalence of general obesity between quartiles of the DASH diet score, $P=0.24$ (a); whereas a marginally significant trend towards decreasing prevalence of central obesity was seen with increasing quartile of the DASH diet score, $P=0.09$ (b)

significantly higher intakes of $\mathrm{Ca}, \mathrm{Mg}, \mathrm{K}$, folate and total dietary fibre (all $P<0.001$ ) and lower intake of $\mathrm{Na}$ $(P=0.07)$ compared with those in the first quartile.

Prevalences of general and central obesity across quartiles of DASH diet scores are shown in Fig. 1. There was no statistically significant difference in the prevalence of general obesity between extreme quartiles of the DASH diet score. Although the prevalence of central obesity was not statistically different across quartiles of DASH diet score, a marginally significant trend towards decreasing prevalence of central obesity was seen with increasing quartile of the DASH diet score $(P=0 \cdot 09)$.

Multivariate-adjusted odds ratios for general and central obesity across quartile categories of DASH diet scores are provided in Table 3. Adherence to the DASH diet was not associated with general obesity in the crude model. After adjustment for age and energy intake and other confounding factors in model II, the DASH diet was not significantly associated with obesity. However, when the models were further controlled for dietary factors, a significant inverse association was seen between the DASH diet and general obesity; so that those in the fourth quartile of DASH score were $71 \%$ less likely to have general obesity compared with those in the first quartile. Consumption of the DASH diet was not significantly associated with central obesity in the crude model. However, after adjustment for age and energy intake, the association became significant (fourth $v$. first quartile: $\mathrm{OR}=0 \cdot 37 ; 95 \%$ CI $0 \cdot 14,0 \cdot 96$ ). Further controlling for potential confounders attenuated the association; so that after inclusion of potential confounders and dietary variables in the model, the association was no longer significant.

\section{Discussion}

We found that adherence to the DASH diet and prevalence of general obesity were inversely related. This association remained statistically significant even after adjustment for potential confounding factors. However, we failed to find a significant association between adherence to the DASH diet and central obesity. To our knowledge, the present study is the first observational one investigating the association between adherence to the DASH diet and risk of general and central obesity in a Middle Eastern population.

Earlier studies have shown that obesity, in particular central obesity, is a contributing factor for several metabolic conditions including heart events and atherosclerosis. In recent decades, obesity has become an epidemic worldwide $^{(1)}$. In line with increasing prevalence of obesity around the world, the rate is increasing also in Middle Eastern countries; so that more than two-thirds of adult women in this region are centrally obese ${ }^{(9)}$. Diet has long been known to contribute to the obesity epidemic. Several studies have shown that healthy dietary patterns are associated with better weight control ${ }^{(24)}$. Although the association between a posteriori-defined dietary patterns and obesity has been reported extensively ${ }^{(27)}$, few data are available investigating the association between a priori-defined dietary patterns and risk of general and central obesity, particularly in Middle Eastern populations ${ }^{(24)}$.

Several cross-sectional and prospective studies as well as clinical trials have indicated the beneficial effects of the DASH diet on metabolic health ${ }^{(17,21)}$. Nearly all existing evidence has been obtained among selected groups of 
Table 3 Multivariate adjusted odds ratios for general and central obesity according to quartile of Dietary Approaches to Stop Hypertension (DASH) diet score among female nurses ( $n$ 293) aged $>30$ years in Isfahan, Iran

\begin{tabular}{|c|c|c|c|c|c|c|c|c|}
\hline & \multicolumn{7}{|c|}{ Quartile of DASH score } & \multirow[b]{3}{*}{$P$ trend } \\
\hline & \multirow[t]{2}{*}{ Q1 } & \multicolumn{2}{|c|}{ Q2 } & \multicolumn{2}{|c|}{ Q3 } & \multicolumn{2}{|c|}{ Q4 } & \\
\hline & & OR & $95 \% \mathrm{Cl}$ & OR & $95 \% \mathrm{Cl}$ & OR & $95 \% \mathrm{Cl}$ & \\
\hline \multicolumn{9}{|c|}{ General obesity $\dagger$} \\
\hline Crude & 1.00 & 0.74 & $0.38,1.42$ & 1.52 & $0.76,3.04$ & 0.85 & $0.45,1.62$ & 0.57 \\
\hline Model I & 1.00 & 0.64 & $0.31,1.29$ & 1.26 & $0.61,2.63$ & 0.68 & $0.34,1.33$ & 0.92 \\
\hline Model II§ & 1.00 & 1.34 & $0.45,3.97$ & $2 \cdot 24$ & $0.73,6.89$ & 0.43 & $0.13,1.34$ & 0.38 \\
\hline Model IIIII & 1.00 & 0.91 & $0.31,2.63$ & 1.71 & $0.54,5.32$ & 0.29 & $0.09,0.97$ & 0.17 \\
\hline \multicolumn{9}{|c|}{ Central obesity } \\
\hline Crude & 1.00 & 0.75 & $0.32,1.71$ & 1.58 & $0.70,3.55$ & 0.50 & $0.20,1.24$ & 0.31 \\
\hline Model I & 1.00 & 0.80 & $0.32,1.96$ & 1.22 & $0.51,2.95$ & 0.37 & $0.14,0.96$ & 0.15 \\
\hline Model II & 1.00 & 2.06 & $0.25,17.01$ & $3 \cdot 29$ & $0.49,21.83$ & 0.31 & $0.04,2.35$ & 0.66 \\
\hline Model III & 1.00 & $3 \cdot 25$ & $0.14,73.67$ & 3.50 & $0.24,51 \cdot 11$ & 0.20 & $0.01,4.33$ & 0.50 \\
\hline
\end{tabular}

MET, metabolic equivalent of task.

Significant odds ratios and $95 \%$ confidence intervals are shown in bold font.

*Mantel-Haenszel extension $\chi^{2}$ test.

tGeneral obesity was defined as $\mathrm{BMI} \geq 25 \mathrm{~kg} / \mathrm{m}^{2}$

¥Model I: adjusted for age and energy intake.

§Model II: additionally adjusted for physical activity (MET-h/week), current corticosteroid use (yes or no), current oral contraceptive pill use (yes or no), menopausal status (yes or no), marital status (married/not married) and socio-economic status (categorical).

IIModel III: further adjusted for intakes of refined grains, sweets, white meat, oils, high-fat dairy and eggs.

ๆCentral obesity was defined as waist circumference $\geq 88 \mathrm{~cm}$.

people, such as diabetic patients. The findings from such studies cannot be easily extrapolated to the general population. Furthermore, the effect of the DASH diet on central obesity has not been given adequate attention so far. In the present study we found an inverse relationship between adherence to the DASH diet and risk of general obesity. This finding is consistent with those reported previously. Folsom et al. ${ }^{(28)}$ found that greater adherence to the DASH diet was associated with lower mean values of waist-to-hip ratio and BMI among women initially aged 55-69 years. Also, another study among Swedish women reported that following a healthy dietary pattern characterized by high intakes of vegetables, fruit, whole grains, fish and legumes, in combination with moderate alcohol consumption, was associated with decreased risk of myocardial infarction ${ }^{(29)}$. Similar findings were reported for men ${ }^{(30)}$ and diabetic youth ${ }^{(16)}$. However, in another study among diabetic patients, the investigators failed to find any significant relationship between DASH diet adherence and $\mathrm{BMI}^{(15)}$. Different findings might be explained by the different definition of the DASH diet across studies as well as the sample size, study population and validity of the dietary assessment tools. While Günther et $a l^{(15)}$ used fats and oils consumption as well as total grains and total dairy intake in the construction of the DASH diet scores, in the present study we did not use fats and oils but used whole grains and low-fat dairy in our score construction. In addition, while for the determination of odds ratios we adjusted for different dietary factors that were not included in the DASH score, such adjustments were not done in previous studies.

The biological mechanisms through which the DASH diet exerts its protective effects are not fully understood yet. It seems that the high contents of dietary fibre, folate, $\mathrm{K}$, vitamin $\mathrm{C}$, flavonoids, carotenoids and phytochemicals in the DASH diet might be responsible for its beneficial health effects ${ }^{(31)}$. The DASH diet contains a large amount of dairy, which has apparently been inversely related to obesity $^{(32)}$. Dairy's Ca has been shown to play an important role in this mechanism. In addition, the DASH diet is a low-glycaemic-index and low-energy-density diet. The protective association of diets with low glycaemic index as well as low energy density on obesity was reported before $^{(33)}$. Such diets stimulate satiety and would in turn result in decreased food intake ${ }^{(33,34)}$. The fibre content of the DASH diet might also provide an additional explanation for its beneficial effects on obesity ${ }^{(35)}$. Dietary fat is believed to affect different gut peptides and adipocytokines that are involved in the pathophysiology of obesity $^{(36)}$. It is possible that the influence of fibre intake on insulin secretion or stimulation of incretin hormones, such as glucagon-like peptide-1, favours reduced adiposity ${ }^{(37)}$. Another possible explanation is that high-fibre diets may alter hypothalamic-pituitary-adrenal activity that may consequently lead to reduced central body fat accumulation ${ }^{(38)}$.

Several points should be considered while interpreting our findings. Due to the cross-sectional nature of the current study, a causal relationship cannot be inferred. The use of an FFQ for assessing dietary intakes would be a source of some sorts of misclassification bias. The scoring criteria that we used in the current study intended to assess adherence to the DASH diet might be different from those used in clinical trials. In addition, our meal-based FFQ is not designed to provide precise assessment of $\mathrm{Na}$ intake, which is a major component of the DASH diet. Therefore, accurate cut-offs cannot be determined for the sodium 
component of the DASH score. We adjusted the association between DASH diet and obesity for several confounders, but residual confounding cannot be excluded. We measured waist circumference at the point of noticeable waist narrowing, which may have resulted in lower waist measures than the values that might be obtained using other common sites of measurement. This location of waist measuring might also have resulted in some measurement errors, because each person would have a different area of the abdomen that is the narrowest part. Such potential source of error is particularly important for the present study that aims to evaluate the Middle Eastern pattern of obesity whose main characteristic is central obesity and enlarged waist circumference. Moreover, the study was conducted among women and the findings cannot be extrapolated to the entire population.

\section{Conclusion}

In conclusion, we found that adherence to the DASH diet was inversely associated with general, not central obesity. Prospective cohort studies are required to confirm these findings.

\section{Acknowledgements}

Acknowledgements: The authors would like to thank the Board of Directors, Isfahan Nursing Organization for their great cooperation in conducting this study. They also appreciate the cooperation received from Samira Mahdavi, Maryam Pozveh, Marzie Heidari, Shokouh Onvani and Simin Shahvazi in data collection. The authors are thankful to the staff of the selected hospitals who took part in the current study. Financial support: The study was supported by the Food Security Research Center (FSRC), Isfahan University of Medical Sciences, Isfahan, Iran in conjunction with Lorestan University of Medical Sciences, Khorramabad, Iran. The funders had no contribution in the design, conducting, writing, analysis and final approval of the manuscript. Conflict of interest: None. Authorship: F.B. and A.E. contributed in conception, design, statistical analysis and drafting of the manuscript. E.F., A.H.K. and A.Y. contributed in data collection and manuscript drafting. A.E. supervised the study. All authors read and approved the final version of the paper. Ethics of buman subject participation: The Regional Biomedical Ethics Committee affiliated to Isfahan University of Medical Sciences approved the study protocol. Informed written consent was obtained from each participant.

\section{References}

1. Caballero B (2007) The global epidemic of obesity: an overview. Epidemiol Rev 29, 1-5.
2. Wagner KH \& Brath H (2012) A global view on the development of non communicable diseases. Prev Med 54, Suppl., S38-S41.

3. Kelly T, Yang W, Chen CS et al. (2008) Global burden of obesity in 2005 and projections to 2030. Int J Obes (Lond) 32, 1431-1437.

4. Ahmad J, Ahmed F, Siddiqui MA et al. (2006) Inflammation, insulin resistance and carotid IMT in first-degree relatives of north Indian type 2 diabetic subjects. Diabetes Res Clin Pract 73, 205-210.

5. Seidell JC, Verschuren WM, van Leer EM et al. (1996) Overweight, underweight, and mortality: a prospective study of 48287 men and women. Arch Intern Med 156, 958-963.

6. Misra A \& Khurana L (2008) Obesity and the metabolic syndrome in developing countries. J Clin Endocrinol Metab 93, 11 Suppl. 1, S9-S30.

7. Popkin BM \& Slining MM (2013) New dynamics in global obesity facing low- and middle-income countries. Obes Rev 14, 11-20.

8. Mehio Sibai A, Nasreddine L, Mokdad AH et al. (2010) Nutrition transition and cardiovascular disease risk factors in Middle East and North Africa countries: reviewing the evidence. Ann Nutr Metab 57, 193-203.

9. Janghorbani M, Amini M, Willett WC et al. (2007) First nationwide survey of prevalence of overweight, underweight, and abdominal obesity in Iranian adults. Obesity (Silver Spring) 15, 2797-2808.

10. Sepanlou SG, Kamangar F, Poustchi H et al. (2010) Reducing the burden of chronic diseases: a neglected agenda in Iranian health care system, requiring a plan for action. Arch Iran Med 13, 340-350.

11. Esmaillzadeh A, Entezari M, Paknahad Z et al. (2008) Identification of diet-disease relations through dietary pattern approach: a review. J Res Med Sci 13, 337-348.

12. Moeller SM, Reedy J, Millen AE et al. (2006) Dietary patterns: challenges and opportunities in dietary patterns research an Experimental Biology workshop. J Am Diet Assoc 107, 1233-1239.

13. US Department of Health and Human Services \& US Department of Agriculture (2005) Dietary Guidelines for Americans 2005. Washington, DC: US Government Printing Office.

14. Svetkey LP, Sacks FM, Obarzanek E et al. (1999) The DASH Diet, Sodium Intake and Blood Pressure Trial (DASHsodium): rationale and design. DASH-Sodium Collaborative Research Group. J Am Diet Assoc 99, 8 Suppl., S96-S104.

15. Günther AL, Liese AD, Bell RA et al. (2009) Association between the dietary approaches to hypertension diet and hypertension in youth with diabetes mellitus. Hypertension 53, 6-12.

16. Liese AD, Nichols M, Sun X et al. (2009) Adherence to the DASH diet is inversely associated with incidence of type 2 diabetes: the insulin resistance atherosclerosis study. Diabetes Care 32, 1434-1436.

17. Azadbakht L, Mirmiran P, Esmaillzadeh A et al. (2005) Beneficial effects of a Dietary Approaches to Stop Hypertension eating plan on features of the metabolic syndrome. Diabetes Care 28, 2823-2831.

18. Blumenthal JA, Babyak MA, Hinderliter A et al. (2010) Effects of the DASH diet alone and in combination with exercise and weight loss on blood pressure and cardiovascular biomarkers in men and women with high blood pressure: the ENCORE study. Arch Intern Med 170, 126-135.

19. Fung TT, Chiuve SE, McCullough ML et al. (2008) Adherence to a DASH-style diet and risk of coronary heart disease and stroke in women. Arch Intern Med 168, 713-720.

20. de Koning L, Chiuve SE, Fung TT et al. (2011) Diet-quality scores and the risk of type 2 diabetes in men. Diabetes Care 34, 1150-1156. 
21. Azadbakht L, Fard NR, Karimi M et al. (2011) Effects of the Dietary Approaches to Stop Hypertension (DASH) eating plan on cardiovascular risks among type 2 diabetic patients: a randomized cross-over clinical trial. Diabetes Care 34, $55-57$.

22. Moore TJ, Alsabeeh N, Apovian CM et al. (2008) Weight, blood pressure, and dietary benefits after 12 months of a Web-based Nutrition Education Program (DASH for health): longitudinal observational study. J Med Internet Res 10, e 52.

23. Miller PE, Cross AJ, Subar AF et al. (2013) Comparison of 4 established DASH diet indexes: examining associations of index scores and colorectal cancer. Am J Clin Nutr 98, 794-803.

24. Esmaillzadeh A \& Azadbakht L (2008) Major dietary patterns in relation to general obesity and central adiposity among Iranian women. J Nutr 138, 358-363.

25. Keshteli AH, Esmaillzadeh A, Rajaei S et al. (2014) A dishbased semi-quantitative food frequency questionnaire for assessment of dietary intakes in epidemiologic studies in Iran: design and development. Int J Prev Med 5, 29-36.

26. Booth M (2000) Assessment of physical activity: an international perspective. Res Q Exerc Sport 71, 114-120.

27. Carrera PM, Gao X \& Tucker KL (2007) A study of dietary patterns in the Mexican-American population and their association with obesity. J Am Diet Assoc 107, 1735-1742.

28. Folsom AR, Parker ED \& Harnack LJ (2007) Degree of concordance with DASH diet guidelines and incidence of hypertension and fatal cardiovascular disease. Am J Hypertens 20, 225-232.

29. Kesson A, Weismayer C, Newby PK et al. (2007) Combined effect of low-risk dietary and lifestyle behaviors in primary prevention of myocardial infarction in women. Arch Intern Med 167, 2122-2127.
30. Levitan EB, Wolk A \& Mittleman MA (2009) Relation of consistency with the dietary approaches to stop hypertension diet and incidence of heart failure in men aged 45 to 79 years. Am J Cardiol 104, 1416-1420.

31. Most MM (2004) Estimated phytochemical content of the Dietary Approaches to Stop Hypertension (DASH) diet is higher than in the control study diet. J Am Diet Assoc 104, $1725-1727$.

32. Azadbakht L, Mirmiran P, Esmaillzadeh A et al. (2005) Dairy consumption is inversely associated with the prevalence of the metabolic syndrome in Tehranian adults. Am J Clin Nutr 82, 523-530.

33. Schwingshackl L \& Hoffmann G (2013) Long-term effects of low glycemic index/load vs. high glycemic index/load diets on parameters of obesity and obesity-associated risks: a systematic review and meta-analysis. Nutr Metab Cardiovasc Dis 23, 699-706.

34. Mendoza JA, Drewnowski A \& Christakis DA (2007) Dietary energy density is associated with obesity and the metabolic syndrome in US adults. Diabetes Care 30, 974-979.

35. Lindström J, Peltonen M, Eriksson JG et al. (2006) Highfibre, low-fat diet predicts long-term weight loss and decreased type 2 diabetes risk: the Finnish Diabetes Prevention Study. Diabetologia 49, 912-920.

36. Sánchez D, Miguel M \& Aleixandre A (2012) Dietary fiber, gut peptides, and adipocytokines. J Med Food 15, 223-230.

37. McKeown NM, Yoshida M, Shea MK et al. (2009) Wholegrain intake and cereal fiber are associated with lower abdominal adiposity in older adults. J Nutr 139, 1950-1955.

38. Vicennati V \& Pasquali R (2000) Abnormalities of the hypothalamic-pituitary-adrenal axis in nondepressed women with abdominal obesity and relations with insulin resistance: evidence for a central and a peripheral alteration. J Clin Endocrinol Metab 85, 4093-4098. 